\title{
Romantic Attraction and Substance Use in 15-Year-Old Adolescents from Eight European Countries
}

\author{
András Költót ${ }^{1,2, *(\mathbb{D}}$, Alina Cosma $\left.{ }^{3}{ }^{(}\right)$, Honor Young ${ }^{4}$, Nathalie Moreau ${ }^{5}$, Daryna Pavlova ${ }^{6}$, \\ Riki Tesler ${ }^{7}$, Einar B. Thorsteinsson ${ }^{8}{ }^{\mathbb{C}}$, Alessio Vieno ${ }^{9}$, Elizabeth M. Saewyc ${ }^{10}$ \\ and Saoirse Nic Gabhainn ${ }^{1}$ \\ 1 Health Promotion Research Centre, School of Health Sciences, National University of Ireland Galway, \\ University Road, H91 TK33 Galway, Ireland \\ 2 Institute of Psychology, ELTE Eötvös Loránd University, Izabella utca 46, 1064 Budapest, Hungary \\ 3 Department of Interdisciplinary Social Science, Faculty of Social and Behavioural Sciences, Utrecht \\ University, P.O. Box 80.140, 3508 TC Utrecht, The Netherlands \\ 4 Centre for the Development and Evaluation of Complex Interventions for Public Health Improvement, \\ Cardiff School of Social Sciences, Cardiff University, 1-3 Museum Place, CF10 3BD Cardiff, UK \\ 5 Independent Researcher, 1180 Brussels, Belgium \\ 6 Department for Monitoring and Evaluation of Social Projects, Ukrainian Institute for Social Research after \\ Oleksandr Yaremenko, 26 Panasa Myrnogo Str., Of. 211, 01011 Kyiv, Ukraine \\ 7 The Department of Health Systems Management, Ariel University, Ramat HaGolan St 65, 40700 Ariel, Israel \\ 8 School of Psychology, Faculty of Medicine and Health, University of New England, \\ Armidale, NSW 2351, Australia \\ 9 Department of Developmental and Social Psychology, University of Padova, 8 via Venezia, \\ 35131 Padova, Italy \\ 10 Stigma and Resilience Among Vulnerable Youth Centre (SARAVYC), School of Nursing, University of British \\ Columbia, T222-2211 Wesbrook Mall, Vancouver, BC V6T 2B5, Canada \\ * Correspondence: andras.kolto@nuigalway.ie
}

Received: 30 July 2019; Accepted: 19 August 2019; Published: 23 August 2019

\begin{abstract}
Sexual minority youth are at higher risk of substance use than heterosexual youth. However, most evidence in this area is from North America, and it is unclear whether the findings can be generalized to other cultures and countries. In this investigation, we used data from the 2014 Health Behaviour in School-aged Children (HBSC) study to compare substance use in same- and both-gender attracted 15-year-old adolescents from eight European countries $(n=14,545)$ to that of their peers who reported opposite-gender attraction or have not been romantically attracted to anyone. Both-gender attracted, and to a lesser extent, same-gender attracted adolescents were significantly more likely to smoke cigarettes, consume alcohol, get drunk and use cannabis, or be involved in multiple substance use in the last 30 days compared to their opposite-gender attracted peers. Those adolescents who have not been in love had significantly lower odds for substance use than all other youth. The pattern of results remained the same after adjusting for country, gender and family affluence. These findings are compatible with the minority stress and romantic stress theories. They suggest that sexual minority stigma (and love on its own) may contribute to higher substance use among adolescents in European countries.
\end{abstract}

Keywords: adolescents; romantic attraction; same-gender attraction; both-gender attraction; sexual minority youth; substance use; alcohol consumption; drunkenness; tobacco; cannabis; HBSC 


\section{Introduction}

Many young people who identify as Lesbian, Gay, Bisexual (LGB), other sexual or gender minority (for example Queer, Transgender or Intersex), or report being attracted to same- or both-gender partners, have poorer health than their peers who identify as heterosexual, cisgender or as exclusively attracted to members of the opposite gender [1,2]. The studies show a large variation in the use of (biological) sex or (socially constructed) gender. They also employ various sexual identity terms or classify youth based on other dimensions of sexual orientation, such as gender of sexual or love partner(s). In this study, we use the term 'gender' to describe whether the respondents identified themselves as boys or girls. The 'sexual minority youth' (SMY) term is used, as this is the most inclusive, unless we refer to studies that used more specific terminology (such as LGB).

Extensive research indicates that SMY are more likely to engage in substance use [3,4]. However, the validity of the evidence is limited by the fact that most investigations have been conducted in North America. There are just a few sporadic observations from other countries, and cross-cultural comparisons are largely missing. This study aimed to describe and compare substance use frequency across patterns of romantic attraction, in nationally representative samples of 15 -year-old adolescents from eight European countries and regions with various geographical location, history, and levels of tolerance towards sexual minorities.

\subsection{Tobacco Use}

While LGB youths appear to start smoking at a later age than the general population, compared to their heterosexual peers they are significantly more likely to use various (and multiple) tobacco products, as well as to report smoking in the past month, or being current smokers [5-8]. Smoking patterns were influenced by sexual identity, gender, race or ethnicity, and their interactions [8-10]. In some studies, significant differences were found between sexual minority girls and boys, or bisexual youths and those identifying as lesbian or gay. Such findings indicate the importance of mapping the relative risk of SMY boys and girls separately.

Cross-sectional and longitudinal studies have concluded that psychological distress in SMY was associated with smoking [11,12]. Sexual minority adolescents were significantly more likely to report smoking in the past year compared to heterosexual youth in a large-sample U.S. national prospective cohort study, after adjusting for gender, age, race/ethnicity, and family income. However, SMY youth living in states where the social environment was less stigmatizing toward LGB people had a significantly lower relative risk for smoking than those who lived in a state imposing stronger structural stigma. The stigmatizing environment did not have a differential effect on heterosexual youth [13]. This indicates that besides micro-environmental influences, macro-level societal indicators may also be associated with substance use in SMY. Therefore, it is important to investigate cross-cultural variations in the associations between sexual minority status and substance use or other risk behaviors, ideally including sexual minority young people from various countries and cultures.

\subsection{Alcohol Consumption and Drunkenness}

Sexual minority adolescents are more likely than heterosexual youth to drink alcohol and get drunk $[3,14]$. LGB young people report earlier alcohol initiation and sharper drinking trajectories into adulthood than heterosexual youth [15]. The experience of sexual minority belonging in adolescence may shape alcohol-related behaviors in later age [16]. Some argue that consuming any amount of alcohol and excessive drinking (i.e., heavy episodic drinking) may diverge, for instance for cultural reasons [17], therefore they should be examined separately.

While there was a general decline in adolescent alcohol use in the United States and Europe over the last decade [18], the alcohol-related disparities between heterosexual and SMY have remained stable or even widened [19]. More recent findings demonstrate that pluri-sexual males (having both-gender sexual partners or identifying as bisexual) have higher risk for earlier onset and persistent use of 
alcohol than those with monosexual behavior (having exclusively opposite- or same-gender partners) or identifying as heterosexual or gay [20].

\subsection{Cannabis Use}

SMY are at an increased risk of cannabis use [21-23]. A systematic review of Canadian studies revealed that cannabis use is consistently higher in SMY than in heterosexual respondents, with sexual minority young men and bisexual youth having greater risk than young women or those identifying as lesbian or gay [24]. Furthermore, trends observed in cannabis use in SMY are similar to those in alcohol and tobacco use. Between 1999 and 2013, a population-based US study found that while cannabis use had decreased overall, the disparities between some SMY subgroups (in lesbian and bisexual females) and their heterosexual counterparts remained stable [25].

Potential explanatory pathways for the association between SMY status and cannabis use are comparable to those of alcohol and tobacco use. Internalized homophobia and community connectedness were both positively associated with cannabis use in LGB young people [26]. These effects may be attributed to minority stress, and greater community connectedness may be associated with greater conformity to social norms within the LGB community that are more permissive toward substance use.

\subsection{Minority and Romantic Stress: Explanation for Different Types and Combinations of Substance Use?}

In addition to the single substance studies cited above, there is a large corpus of evidence on multiple or poly-substance use and the association with mental health outcomes in SMY. These studies examine alcohol, tobacco and cannabis or other drugs [20,25,27], alcohol and cigarettes [28], drugs and alcohol [29,30], tobacco, methamphetamine use and suicidal ideation [31], or meta-analyses where different types of substance use were pooled [3,4].

In a systematic review of 18 studies [3], it was found that LGB youth were around three times more likely, compared to their heterosexual peers, to be involved in any type of substance use. The effects were larger in bisexual compared to lesbian/gay young people, and in females compared to males. When one large-effect size study was removed from the pool, no significant differences were observed between studies conducted in the United States or elsewhere, which suggests that the disparity may be universal across different countries and cultures. Another systematic review of 12 studies revealed that the strongest risk factors for substance use (smoking cigarettes, consuming alcohol, cannabis, cocaine and ecstasy) in SMY were LGB-related or general victimization, lack of supportive environments, psychological stress, internalizing/externalizing behaviors, negative responses to coming out, and housing status [4].

The disparities between SMY and heterosexual youth's substance use can be explained by the minority stress theory which argues that experiences of discrimination, victimization, and stigma are prevalent due to a pervasive homophobic culture [32,33]. The existing literature points out that sexual orientation-based bullying and harassment at school contributes to SMY disparities in all forms of substance use. Hatzenbuehler's [34] extension of minority stress suggests that due to stigmatization, sexual (and gender) minority individuals experience chronic stress, which in the long term may lead to deficits in emotion regulation and negative affect. To cope with these, sexual minority individuals may turn to alcohol (and other substance) use [4,12,35].

Consistent with the ecological framework provided by minority stress theory, it is important to examine factors that may predict substance use, particularly societal attitudes and policies regarding sexual minority communities and individuals. Although attitudes toward sexual minorities also are changing in several parts of the world, there are still many countries with strong anti-LGB policies or cultural norms. The negative effects of stigma and discrimination on sexual minority individuals' health, including minority stress, depression, and fear of seeking help are well-documented. However, most of the evidence is from North America [36]. The question remains whether these findings can be generalized to other countries and cultures (i.e., in the European region), given the large variation 
in societal attitudes, tolerance and acceptance towards gender and sexual minority individuals and issues within Europe [37,38].

The countries involved in our study represent large variation both geographically (from Iceland to North Macedonia), historically (from traditionally Capitalist countries such as Belgium, England, France and Switzerland to post-Communist countries as Bulgaria and Hungary), and in terms of tolerance towards sexual minorities. The latter can be demonstrated by the International Lesbian Gay, Bisexual, Trans and Intersex Association's (https://www.ilga-europe.org/rainboweurope) Rainbow Score, a composite measure reflecting the legal situation and acceptance of gender and sexual minorities in different countries, ranging from 0 (gross violations of human rights) to 100 (full respect of human rights, full equality between sexual and gender minority and heterosexual and cisgender individuals). In the eight countries or regions involved in the present study, the Rainbow Score in 2014, when the data were collected, ranged from $13 \%$ in North Macedonia to $82 \%$ in the United Kingdom [39].

In a nine-country investigation of substance use in 16-35-year-old LGB and heterosexual individuals, Demant and colleagues [40] argue that cross-cultural comparisons in this area are important because cultural norms and attitudes towards both substances and sexual minority identities show considerable variations across countries with liberal versus more conservative policies and regulations. Until such studies are replicated, we cannot conclude that higher frequency of substance use in SMY is a universal phenomenon. Therefore, in this study we aimed to explore the associations between SMY and different substance use behaviors across different European countries and regions.

Another potential explanation, partly overlapping with the minority stress model, is that love, irrespective of the gender of the partner(s) with whom a young person is in love with, may be associated with stress on its own. Indeed, a cross-cultural study conducted in 17 countries found that adolescents experienced stress related the romantic relationships, especially in Mid- and South-European countries. Overall, around $20 \%$ of the adolescents used externalizing coping strategies, such as alcohol and drug use, to cope with these stressors [41]. This prompts the notion that maybe not just same-or both-gender attracted adolescents may be at elevated risk of substance use, but anyone who are in love may be at higher risk than those who are not being in love.

\subsection{Dimensions of Sexual Orientation}

The number of young people with same-gender attractions far exceeds those who engage in same-gender sexual behavior or who identify as lesbian, gay or bisexual. This is consistent with findings from large-scale nationally representative studies with adults, where the proportion of individuals with same- or both-gender attraction was much larger than those who identified as LGB [42]. A population-based study in the United Kingdom demonstrated substantial diversity between identity, behavior and attraction in sexual minority adults [43]. Studies have also varied on how they categorized SMY (identity, behavior or attraction), and whether they separated mono- and plurisexual youth. In one study, respondents as young as 9-10 years old were asked whether they consider themselves to be lesbian, gay or bisexual [44]. While acknowledging the importance of all dimensions of sexual orientation, we argue that asking whether adolescents are attracted to girls, boys or both-gender partners may be easier for young people to answer, be more accurate, and can be used to subsequently categorize SMY based on same- or both-gender romantic attraction. This approach may be developmentally more appropriate than employing the identity labels of sexual orientation [45]. Relying on sexual identity as a classifier for SMY may 'mask' or eliminate those young people who are still exploring their sexuality, have same- or both-gender attraction, but do not identify as LGB.

Health disparities in SMY can be found when respondents are classified by same- or both-gender romantic attraction. In a nationally representative study of U.S. adolescents [46], boys romantically attracted to both-gender partners smoked more cigarettes, were more likely to have consumed alcohol while being alone, to have been drunk, and to use illegal drugs (including cannabis) compared to those who had been attracted to the opposite gender. Girls attracted to their same- or both-gender peers were more likely to smoke cigarettes, have been drunk, and have used cannabis or other drugs 
compared to opposite-gender attracted females. However, their conclusion was that SMY or certain subgroups within this category had a greater risk of substance use than heterosexually identifying or exclusively opposite-gender attracted youth. Therefore, in the current study we anticipate finding significant gender differences in the associations being investigated.

Another U.S. adolescent study demonstrated that sexual identity (i.e., defining oneself as LGB) and sexual behavior (i.e., having exclusively same- or both-gender partners) explained unique and significant sources of variability in tobacco and methamphetamine use and suicidal ideation [31]. In another investigation, same- and both-sex romantic attraction and romantic relationship status were associated with various risk behaviors such as the number of cigarettes smoked in the past month, being drunk in the past year, and cannabis or other drug use [46]. When adolescents were categorized into SMY based not on their identity but either on a history of same-gender attraction or sexual behavior, a sharper increase was observed in their cigarette and cannabis use than in those adolescents with heterosexual identity (or opposite-gender attraction or behavior) [47]. These findings demonstrate that apart from identity, other dimensions of sexual orientation (i.e., behavior or romantic attraction) may also be associated with higher incidence of risk behaviors.

Based on these considerations and empirical evidence, in the present study, romantic attraction will be used to classify sexual minority adolescents and separate adolescents reporting being in love with any gender partners from those who have not been in love.

\subsection{Aims of the Present Study}

We aimed to describe and compare substance use frequency across patterns of romantic attraction, in nationally representative samples of 15-year-old adolescents from eight European countries and regions.

Romantic attraction was operationalized by an item on whether the respondent had already been in love, and if yes, whether the partner who they felt love for was a girl(s), boy(s), or both- a boy and a girl [45]. This approach is in line with the notion that romantic attraction and love are conditional to each other [48]. The responses, combined with the gender of the respondent, enabled us to categorize opposite-, same-, or both-gender attracted respondents, those who have not been attracted to anyone, or who have not responded to the love item. Contrary to most studies that concentrate on those with any type of attraction or sexual identity, we also measured the prevalence of substance use in those who reported not having been in love or who did not respond to this item. Based on previous findings from the literature, we hypothesized that same- and both-gender attracted young people will have significantly higher odds of cigarette smoking, drinking alcohol, being drunk, and cannabis use than their opposite-gender attracted peers or those who reported not having been in love. Our other hypothesis is that those young people who report being in love (with any gender partners) will have higher odds of substance use than those who have not been in love. We anticipated that despite cultural differences in the prevalence of these risk behaviors, higher incidence of substance use will be found in same- and both-gender attracted young people (and those who report being in love) across different countries and regions. Given the differences between sexual minority boys and girls found in many studies, analyses were stratified for gender.

An additional aim was to assess involvement of SMY in multiple risk behaviors (any two or all three of cigarette smoking, alcohol consumption, or cannabis use in the last 30 days). We hypothesized that youth reporting attraction to same- or both-gender partners will be more likely to be involved in using more than one type of substances than those who are exclusively attracted to opposite-gender partners or reported not having been in love.

\section{Materials and Methods}

Data was collected within the 2014 survey round of the Health Behaviour in School-aged Children (HBSC) study, a World Health Organization collaborative cross-national epidemiological study. The HBSC study investigates health-related behaviors and related psychosocial contextual 
factors in nationally representative samples of 11-, 13- and 15-year-old school children, in four-year study cycles across more than forty countries, covering the geographical areas of Europe, North America, and former Soviet Republics. There were 42 countries that collected data as part of the HBSC international survey in 2014. Out of these, data from eight countries and regions are featured in this paper. A detailed description of HBSC methodology is provided by Inchley et al. [49] and Currie et al. [50]. In HBSC, a survey questionnaire is employed containing (1) items administered in each participating countries in the same format ('mandatory' items), (2) items following the same format, but the national team decides if they will be administered in the questionnaire ('optional' items) and (3) items that are relevant for the health of young people in the given country ('national' items). In the present study, substance use was monitored using mandatory items, while romantic attraction was measured by an optional item. As such, the measure of romantic attraction was included in the national surveys if the research team in the given country or region considered investigating the health of sexual minority youth substantially important. The methodology used by HBSC is described at http://www.hbsc.org/methods/index.html, and details on data access are provided at https://www.uib.no/en/hbscdata.

\subsection{Sample}

Schoolchildren in the 15-year-old age group from eight countries and regions (French Belgium, Bulgaria, Switzerland, England, France, Hungary, Iceland, and North Macedonia) where the national HBSC Research Team included the measure on romantic attraction (see below, Section 2.2) in their national survey. The raw sample contained data from 14,545 respondents (mean age: 15.55 years, $S D=0.33$, range: $14.58-16.50$, percentage girls: 49.8 ). Listwise deletion was employed (for all predictor, outcome and sociodemographic control variables) to determine the number of respondents featured in the final statistical models. There were 13,504 respondents $(92.8 \%)$ in the cigarette smoking model; 13,440 respondents $(92.4 \%)$ in the alcohol consumption model; 13,471 respondents $(92.6 \%)$ in the drunkenness model; 12,109 respondents (83.3\%) in the cannabis use model; and 13,580 respondents $(93.4 \%)$ in the multiple substance use model. The characteristics of the sample are displayed in Table 1. Since the most respondents were featured in the multiple substance use model, the distribution of the Love item and sociodemographic variables are given for this headcount $(n=13,580)$.

\subsection{Ethical Considerations}

In each country, the HBSC research team sought ethical approval from local or national higher education or health authorities: Boards of School Networks of the Brussel-Wallonia Federation (French Belgium), Ministry of Education and Science (Bulgaria), University of Lausanne, Cantonal Commission for Ethics for the Research on Human Beings (Switzerland), University of Hertfordshire, Ethics Committee for Studies Involving Human Participants (England), Ministry of Education and the French National Commission of Computer Science and Freedom (France), Scientific and Research Ethics Committee of the Medical Research Council (Hungary), Icelandic Data Committee (Iceland), and the Ministry for Education and Ministry for Health (North Macedonia). In the eight countries and regions involved in this study, pupils (as well as their parents and the schools) gave informed consent to participate in the study. Before administering the questionnaire, respondents were instructed that responding to any question or the whole questionnaire was entirely voluntary, and they could withdraw at any time. The questionnaires were anonymous and treated as confidential. Our research procedures are following the WHO Standards and operational guidance for ethics review of health-related research with human participants (https://www.who.int/ethics/research/en/). 
Table 1. Characteristics of the sample, overall and by romantic attraction $(n=13,580)$.

\begin{tabular}{|c|c|c|c|c|c|c|c|c|c|c|c|c|c|}
\hline & \multicolumn{2}{|c|}{ OVERALL } & \multicolumn{2}{|c|}{ Opposite-Gender Love } & \multicolumn{2}{|c|}{ Same-Gender Love } & \multicolumn{2}{|c|}{ Both-Gender Love } & \multicolumn{2}{|c|}{ Not in Love } & \multicolumn{2}{|c|}{ Not Responding } & \multirow{2}{*}{ Assoc. ${ }^{2}$} \\
\hline & $n$ & $\%$ & $n$ & $\%\left(\right.$ RA) ${ }^{1}$ & $n$ & $\%$ (RA) & $n$ & $\%$ (RA) & $n$ & $\%$ (RA) & $n$ & $\%$ (RA) & \\
\hline \multicolumn{14}{|l|}{ Love } \\
\hline Opposite-sex love & 11,024 & 81.2 & & & & & & & & & & & \\
\hline Same-sex love & 219 & 1.6 & & & & & & & & & & & \\
\hline Both-sex love & 248 & 1.8 & & & & & & & & & & & \\
\hline Not in love & 1756 & 12.9 & & & & & & & & & & & \\
\hline Not responding & 333 & 2.5 & & & & & & & & & & & \\
\hline Belgium (French) & 1779 & 13.1 & 1464 & 13.3 & 27 & 12.3 & 25 & 10.1 & 222 & 12.6 & 41 & 12.3 & $p<0.001$ \\
\hline Bulgaria & 1542 & 11.4 & 1306 & 11.8 & 61 & 27.9 & 38 & 15.3 & 115 & 6.5 & 22 & 6.6 & $V=0.173$ \\
\hline Switzerland & 1692 & 12.5 & 1503 & 13.6 & 9 & 4.1 & 21 & 8.5 & 149 & 8.5 & 10 & 3.0 & \\
\hline England & 1442 & 10.6 & 748 & 6.8 & 23 & 10.5 & 37 & 14.9 & 578 & 32.9 & 56 & 16.8 & \\
\hline France & 1658 & 12.2 & 1365 & 12.4 & 34 & 15.5 & 33 & 13.3 & 207 & 11.8 & 19 & 5.7 & \\
\hline Hungary & 1085 & 8.0 & 853 & 7.7 & 4 & 1.8 & 16 & 6.5 & 129 & 7.3 & 83 & 24.9 & \\
\hline Iceland & 2980 & 21.9 & 2707 & 24.6 & 49 & 22.4 & 57 & 23.0 & 151 & 8.6 & 16 & 4.8 & \\
\hline North Macedonia & 1402 & 10.3 & 1078 & 9.8 & 12 & 5.5 & 21 & 8.5 & 205 & 11.7 & 86 & 25.8 & \\
\hline \multicolumn{14}{|l|}{ Gender } \\
\hline Girl & 6848 & 50.4 & 5402 & 49.0 & 123 & 56.2 & 178 & 71.8 & 994 & 56.6 & 151 & 45.3 & $V=0.080$ \\
\hline \multicolumn{14}{|l|}{ Relative FAS } \\
\hline Lowest 20 percent & 2828 & 20.8 & 2217 & 20.1 & 67 & 30.6 & 63 & 25.4 & 393 & 22.4 & 88 & 26.4 & $p<0.001$, \\
\hline Medium 60 percent & 8127 & 59.8 & 6618 & 60.0 & 113 & 51.6 & 145 & 58.5 & 1070 & 60.9 & 181 & 54.4 & $V=0.037$ \\
\hline Highest 20 percent & 2625 & 19.3 & 2189 & 19.9 & 39 & 17.8 & 40 & 16.1 & 293 & 16.7 & 64 & 19.2 & \\
\hline
\end{tabular}

$1 \%$ (RA): Proportion within the given romantic attraction category. ${ }^{2}$ Assoc.: Association between the given variable and romantic attraction. 


\subsection{Measures}

Romantic attraction was measured by a standardized item "Have you ever been in love with ... ", response options being "A girl or girls", "A boy or boys", "Both girls and boys", "I have never been in love". Girls who reported being in love with boys, and boys who reported being in love with girls were categorized into the opposite-gender love group, while girls who reported being in love with girls and boys reporting love for boys were categorized into the same-gender love group. Respondents reporting being in love with both girls and boys were categorized into the both-gender love group. A fourth group consisted of those respondents reporting having never been in love, while the fifth category included those who did not answer the item. The development and basic descriptive statistics for the question are reported elsewhere [45].

Substance use: Four standardized items were used to measure the frequency of substance use in the last 30 days [50,51]. "On how may days (if any) have you smoked cigarettes (tobacco) in the last 30 days?", "On how many days (if any) have you drunk alcohol in the last 30 days?", "Have you ever taken cannabis (hashish, grass, pot) in the last 30 days?" with response options being "Never", "1-2 days", " $3-5$ days", "6-9 days", "10-19 days", “20-29 days", "30 days (or more)". "Have you ever had so much alcohol that you were really drunk in the last 30 days?", with response options being "Never", "Yes, once", "Yes, 2-3 times", "Yes, 4-10 times", "Yes, more than 10 times". In line with methodological recommendations and reporting practice of the European School Survey Project on Alcohol and Other Drugs (ESPAD) for fifteen-year-olds [51], the four substance use variables were dichotomized into reporting never having used the given substance (never being drunk) versus ever. We created a dichotomous variable to express multiple substance use. If the respondent reported any two of cigarette smoking, alcohol consumption and cannabis use in the last 30 days, they were categorized into ever being involved in multiple substance use.

Gender and age: Respondents were asked to indicate whether they are a boy or a girl, as well as to report their date of birth (month/year).

Socioeconomic status was measured by the Family Affluence Scale (FAS), a six-item composite measure developed by the HBSC network [52-54]. FAS measures material family wealth as an indicator of socio-economic position. It asks about concrete possessions (i.e., number of family cars; computers), characteristics of the home (i.e., having a bedroom for one own; number of bathrooms; owning a dishwasher), and the number of family holidays in the last year. The scores are summed up. The absolute Family Affluence Scale scores $(0=$ lowest affluence, $13=$ highest affluence) were then transformed into a ridit-based trichotomous variable separating children from families within the lowest $20 \%$, the medium $60 \%$, and the highest $20 \%$ affluence categories [49].

\subsection{Statistical Analysis}

Data analysis was carried out in IBM SPSS Statistics for Windows, version 25.0 (IBM Corp., Armonk, NY, USA). First, descriptive analyses were conducted for the overall sample and broken down into categories of romantic attraction. Chi-square tests were computed along with Cramér's $V$ effect sizes to check for potential associations between romantic attraction and the sociodemographic and substance use variables. Uni- and multivariate binary logistic regression models were built to map the odds of substance use in adolescents belonging to other romantic attraction categories, compared to those who reported (exclusively) opposite-gender love.

Univariate models were constructed to obtain crude odds ratios (COR) of substance use in different romantic attraction groups. The reference was the group reporting opposite-gender love. Then country, gender and relative FAS grouping were added to the models to obtain adjusted odds ratios (AOR). French Belgium, boys, and adolescents belonging to the lowest family affluence group were set as reference categories. Multivariate analyses were carried out for the entire sample and stratified for gender. Wald statistics indicated that each predictor variable made a significant contribution to the models $(p \leq 0.04)$. Model fit was examined. In many cases, the Chi-square tests indicated poor fit $(p>0.05)$, which may be a result of the large (overall) sample size and the imbalance between the 
compared subgroup sizes. This does not necessarily mean that the model should be discredited $[55,56]$. No collinearity was observed in the predictor variables. To test the potential confounding effect by interactions between the predictors, we constructed models that included two-way interactions, but these did not improve model fit.

It was at the discretion of the national HBSC teams to decide whether they would weight their data to correct imbalances in the composition of the sample. Data were not weighted if the characteristics of the actual sample corresponded to those of the national sampling frame (e.g., gender or family affluence distribution). The only exception to this was France. It means that from the eight national data sets included in this analysis, weighting was only applied to the French data. Therefore, we have used a weight variable with actual values for the French data and set to 1 for data from other countries.

The HBSC study uses classrooms as sampling units. To check whether cluster-based sampling method impacted the results, we have carried out the analyses using the Complex Samples function in SPSS. Design effects in the multivariate models, indicating the extent to which clustering effect needs to be corrected, were not substantially different from $1(0.98 \leq \mathrm{DEFF} \leq 1.24)$, indicating that clustering had a negligible impact. Therefore, the analyses have not been adjusted for cluster sampling.

\section{Results}

The number of respondents in each substance use group in the binary logistic models was determined by how many answered the given substance use item. As Table 2 shows, most respondents reported on the frequency of smoking item in the last 30 days $(n=13,504)$, while a lower number answered the items on alcohol consumption $(n=13,440)$, drunkenness $(n=13,471)$, and cannabis use $(n=12,109)$. For the multiple substance use model, all responses featured in any two of the single substance use models were collapsed $(n=13,580)$.

\subsection{Love and Sociodemographic Characteristics}

There was a significant association between love and country: $\chi^{2}(28)=1625.87, p<0.001$, but with a low effect size: $V=0.173$ (Table 1). Love was associated with gender of the respondents: $\chi^{2}(4)=87.30$, $p<0.001$, but with a low effect size: $V=0.080$. More girls reported same-gender love than boys, and the difference was even larger in the case of both-gender love. Girls were also more likely than boys to report not having been in love, but they were less likely than boys not to respond to the item. Love was also associated with family affluence: $\chi^{2}(8)=36.46, p<0.001$, but the effect size was negligible: $V=0.037$.

\subsection{Romantic Attraction and Substance Use}

The prevalence of substance use across different attraction patterns is displayed in Table 2. Respondents reporting both-gender love reported the highest prevalence for each substance use: Cigarette smoking (33.6\%), drinking alcohol (51.2\%), being drunk (25.1\%), and cannabis use in the last 30 days $(20.6 \%)$. They also reported the highest rate of engagement in multiple substance use in the last 30 days (30.2\%). Across all these romantic attraction groups, the lowest rates of engagement with substance use (apart from alcohol use) was reported by those who have never been in love. 
Table 2. Frequency of risk behaviors, overall and by romantic attraction.

\begin{tabular}{|c|c|c|c|c|c|c|c|c|c|c|c|c|c|}
\hline & \multicolumn{2}{|c|}{ OVERALL } & \multicolumn{2}{|c|}{ Opposite-Gender Love } & \multicolumn{2}{|c|}{ Same-Gender Love } & \multicolumn{2}{|c|}{ Both-Gender Love } & \multicolumn{2}{|c|}{ Not in Love } & \multicolumn{2}{|c|}{ Not Responding } & \multirow{2}{*}{ Assoc. $^{2}$} \\
\hline & $n$ & $\%$ & $n$ & $\%\left(\right.$ RA) ${ }^{1}$ & $n$ & $\%$ (RA) & $n$ & $\%$ (RA) & $n$ & $\%$ (RA) & $n$ & $\%$ (RA) & \\
\hline Cigarette smoking in the last 30 days & 13,504 & & & & & & & & & & & & \\
\hline No & 11,097 & 82.2 & 8933 & 81.5 & 150 & 69.4 & 164 & 66.4 & 1580 & 90.3 & 270 & 81.1 & $p<0.001$ \\
\hline Yes & 2407 & 17.8 & 2025 & 18.5 & 66 & 30.6 & 83 & 33.6 & 170 & 9.7 & 63 & 18.9 & $V=0.105$ \\
\hline Alcohol consumption in the last 30 days & 13,440 & & & & & & & & & & & & \\
\hline No & 8348 & 62.1 & 6656 & 61.0 & 122 & 55.7 & 120 & 48.8 & 1214 & 70.0 & 236 & 70.7 & $p<0.001$ \\
\hline Yes & 5092 & 37.9 & 4251 & 39.0 & 97 & 44.3 & 126 & 51.2 & 520 & 30.0 & 98 & 29.3 & $V=0.079$ \\
\hline Drunkenness in the last 30 days & 13,471 & & & & & & & & & & & & \\
\hline No & 11,680 & 86.7 & 9463 & 86.5 & 168 & 77.1 & 182 & 74.9 & 1587 & 90.8 & 280 & 87.0 & $p<0.001$ \\
\hline Yes & 1791 & 13.3 & 1477 & 13.5 & 50 & 22.9 & 61 & 25.1 & 161 & 9.2 & 42 & 13.0 & $V=0.073$ \\
\hline Cannabis use in the last 30 days & 12,109 & & & & & & & & & & & & \\
\hline No & 11,159 & 92.2 & 9149 & 92.2 & 165 & 84.2 & 185 & 79.4 & 1522 & 94.5 & 138 & 91.4 & $p<0.001$ \\
\hline Yes & 950 & 7.8 & 770 & 7.8 & 31 & 15.8 & 48 & 20.6 & 88 & 5.5 & 13 & 8.6 & $V=0.083$ \\
\hline Multiple substance use in the last 30 days ${ }^{3}$ & 13,580 & & & & & & & & & & & & \\
\hline No & 11,499 & 84.7 & 9262 & 84.0 & 165 & 75.3 & 173 & 69.8 & 1610 & 91.7 & 289 & 86.8 & $p<0.001$ \\
\hline Yes & 2081 & 15.3 & 1762 & 16.0 & 54 & 24.5 & 75 & 30.2 & 146 & 8.3 & 44 & 13.2 & $\mathrm{~V}=0.097$ \\
\hline
\end{tabular}

$1 \%$ (RA): Proportion within the given romantic attraction category. ${ }^{2}$ Assoc.: Association between the given variable and romantic attraction. ${ }^{3}$ Any two of cigarette smoking, alcohol consumption or cannabis use in the last 30 days. 


\subsection{Cigarette Smoking across Romantic Attraction}

The univariate models indicated that same-gender and both-gender attracted respondents were significantly more likely to smoke in the last 30 days, while those not having been in love were less likely to report this behavior compared to opposite-gender attracted respondents (Table 3). Adjusting the model for country/region, family affluence, and gender did not substantially change the odds (the full model is displayed in supplementary Table S1). Compared to opposite-gender attracted adolescents, those who had been in love with both-gender partners had odds of 2.3, while same-gender attracted had odds of 1.9 for smoking. Those not having been in love had significantly lower odds $(\mathrm{AOR}=0.4)$. Those who did not respond to the love item had statistically similar odds of smoking to those who were opposite-gender attracted. Gender-stratified analyses demonstrated that both-gender attracted boys and girls had somewhat higher odds of smoking ( $\mathrm{AOR}=2.8$ and 2.1, respectively) than the same-gender attracted boys and girls ( $\mathrm{AOR}=2.4$ and 1.6, respectively), but these differences were not statistically different. Those who reported never having been in love were significantly less likely to smoke (boys' $\mathrm{AOR}=0.5$; girls' $\mathrm{AOR}=0.4$ ) compared to opposite-gender attracted youth.

\subsection{Alcohol Consumption across Romantic Attraction}

Same-gender attracted youth did not have higher odds of alcohol consumption in the last 30 days than those reporting love with opposite-gender partners, but both-gender attracted youth had significantly higher odds, whereas those not attracted and non-responders had significantly lower odds (Table 3). The unadjusted model and model adjusted for country/region, family affluence, and gender yielded a similar pattern (the full model can be found in supplementary Table S2). Compared to their opposite-gender attracted peers, those who reported love for both-gender partners had odds of 1.8 for alcohol consumption, while those reporting never having been in love or not responding to the love item had odds of 0.5. However, analyses stratified for gender showed that only same-gender attracted boys ( $\mathrm{AOR}=1.7$ ) and both-gender attracted girls ( $\mathrm{AOR}=2.2$ ) were significantly more likely to have had alcohol in the last 30 days. Both boys and girls who reported never being in love or who did not respond to the item on love had significantly lower odds of alcohol consumption.

\subsection{Drunkenness across Romantic Attraction}

In the univariate model, same- and both-gender attracted youth were significantly more likely to report drunkenness in the last 30 days than opposite-gender attracted respondents, while those who had been in love less likely (Table 3). The same pattern was found in the multivariate model adjusted for country/region, family affluence, and gender (the full model can be found in supplementary Table S3). Compared to opposite-gender attracted youth, those reporting same-gender attraction had of 1.8 times, and both-gender love odds of 2.2 (both significantly higher) for drunkenness, while never attracted $(\mathrm{AOR}=0.5)$ and non-responding youth $(\mathrm{AOR}=0.7)$ had significantly lower odds. Same-gender attracted boys were significantly more likely to report drunkenness ( $\mathrm{AOR}=2.4$ ) but same-gender attracted girls were not $(\mathrm{AOR}=1.4)$. No gender differences were observed in the other groups. 
Table 3. Crude and adjusted odds for the four types of substance use, overall and by gender.

\begin{tabular}{|c|c|c|c|c|c|c|c|c|c|c|c|c|}
\hline & \multicolumn{3}{|c|}{ Univariate Model } & \multicolumn{3}{|c|}{ Multivariate Model (Overall) } & \multicolumn{6}{|c|}{ Multivariate Model Stratified for Gender } \\
\hline & $\mathrm{COR}^{1}$ & $p$ & $(95 \% \mathrm{CI})$ & AOR $^{2}$ & $p$ & $(95 \% \mathrm{CI})$ & AOR & $p$ & $(95 \%$ CI $)$ & AOR & $p$ & $(95 \% \mathrm{CI})$ \\
\hline Cigarettes in the last 30 days & \multicolumn{3}{|c|}{$(n=13,504)$} & \multicolumn{3}{|c|}{$(n=13,504)$} & \multicolumn{3}{|c|}{ Boys $(n=6693)$} & \multicolumn{3}{|c|}{ Girls $(n=6811)$} \\
\hline Opposite-gender love & 1 & & & 1 & & & 1 & & & 1 & & \\
\hline Same-gender love & 2.00 & $<0.001$ & $(1.48-2.69)$ & 1.85 & $<0.001$ & $(1.34-2.55)$ & 2.36 & $<0.001$ & $(1.46-3.82)$ & 1.57 & $<0.001$ & $(1.02-2.41)$ \\
\hline Both-gender love & 2.28 & $<0.001$ & $(1.74-2.99)$ & 2.31 & $<0.001$ & $(1.71-3.13)$ & 2.80 & $<0.001$ & $(1.65-4.75)$ & 2.10 & $<0.001$ & $(1.44-3.04)$ \\
\hline Not in love & 0.47 & $<0.001$ & $(0.40-0.56)$ & 0.44 & $<0.001$ & $(0.37-0.52)$ & 0.53 & $<0.001$ & $(0.41-0.70)$ & 0.38 & $<0.001$ & $(0.30-0.48)$ \\
\hline Not responding & 1.08 & 0.599 & $(0.81-1.43)$ & 0.89 & 0.450 & $(0.67-1.20)$ & 1.12 & 0.584 & $(0.75-1.65)$ & 0.68 & 0.093 & $(0.44-1.07)$ \\
\hline Alcohol in the last 30 days & \multicolumn{3}{|c|}{$(n=13,440)$} & \multicolumn{3}{|c|}{$(n=13,440)$} & \multicolumn{3}{|c|}{ Boys $(n=6693)$} & \multicolumn{3}{|c|}{ Girls $(n=6811)$} \\
\hline Opposite-gender love & 1 & & & 1 & & & 1 & & & 1 & & \\
\hline Same-gender love & 1.27 & 0.093 & $(0.96-1.66)$ & 1.20 & 0.230 & $(0.89-1.63)$ & 1.66 & 0.036 & $(1.03$ & 0.97 & 0.880 & $(0.6$ \\
\hline Both-gender love & 1.67 & $<0.001$ & $(1.29-2.15)$ & 1.80 & $<0.001$ & $(1.33-2.43)$ & 1.08 & 0.784 & $(0.64-1.81)$ & 2.15 & $<0.001$ & $(1.50-3.08)$ \\
\hline Not in love & 0.67 & $<0.001$ & $(0.60-0.75)$ & 0.52 & $<0.001$ & $(0.46-0.59)$ & 0.56 & $<0.001$ & $(0.47-0.67)$ & 0.49 & $<0.001$ & $(0.42-0.58)$ \\
\hline Not responding & 0.66 & 0.001 & $(0.52-0.84)$ & 0.48 & $<0.001$ & $(0.38-0.62)$ & 0.57 & 0.001 & $(0.41-0.79)$ & 0.37 & $<0.001$ & $(0.26-0.56)$ \\
\hline Drunkenness in the last 30 days & \multicolumn{3}{|c|}{$(n=13,471)$} & \multicolumn{3}{|c|}{$(n=13,471)$} & \multicolumn{3}{|c|}{ Boys $(n=6693)$} & \multicolumn{3}{|c|}{ Girls $(n=6811)$} \\
\hline Opposite-gender love & 1 & & & 1 & & & 1 & & & 1 & & \\
\hline Same-gender love & 1.92 & $<0.001$ & $(1.39-2.67)$ & 1.81 & 0.001 & $(1.28-2.55)$ & 2.42 & $<0$. & $(1.4$ & 1.38 & 0.204 & $(0.8$ \\
\hline Both-gender love & 2.20 & $<0.001$ & $(1.63-2.96)$ & 2.19 & $<0.001$ & $(1.59-3.02)$ & 1.93 & 0.016 & $(1.13-3.31)$ & 2.25 & $<0.001$ & $(1.51-3.34)$ \\
\hline Not in love & 0.65 & $<0.001$ & $(0.55-0.77)$ & 0.51 & $<0.001$ & $(0.43-0.61)$ & 0.55 & $<0.001$ & $(0.42-0.72)$ & 0.47 & $<0.001$ & $(0.37-0.60)$ \\
\hline Not responding & 0.98 & .879 & $(0.70-1.36)$ & 0.68 & 0.021 & $(0.48-0.94)$ & 0.71 & 0.120 & $(0.46-1.09)$ & 0.63 & .090 & $(0.37-1.07)$ \\
\hline Cannabis in the last 30 days & \multicolumn{3}{|c|}{$(n=12,109)$} & \multicolumn{3}{|c|}{$(n=12,109)$} & \multicolumn{3}{|c|}{ Boys $(n=5999)$} & \multicolumn{3}{|c|}{ Girls $(n=6110)$} \\
\hline Opposite-gender love & 1 & & & 1 & & & 1 & & & 1 & & \\
\hline Same-gender love & 2.21 & $<0.001$ & $(1.48-3.31)$ & 2.16 & 0.001 & $(1.39-3.36)$ & 2.90 & 0.001 & $(1.57-5.38)$ & 1.62 & 0.142 & $(0.85-3.09)$ \\
\hline Both-gender love & 3.19 & $<0.001$ & $(2.30-4.44)$ & 3.57 & $<0.001$ & $(2.48-5.13)$ & 4.12 & $<0.001$ & $(2.33-7.30)$ & 3.20 & $<0.001$ & $(1.99-5.15)$ \\
\hline Not in love & 0.68 & 0.001 & $(0.54-0.85)$ & 0.62 & $<0.001$ & $(0.48-0.79)$ & 0.71 & 0.048 & $(0.51-1.00)$ & 0.53 & 0.001 & $(0.36-0.77)$ \\
\hline Not responding & 1.18 & 0.581 & $(0.66-2.10)$ & 1.05 & 0.979 & $(0.57-1.93)$ & 1.10 & 0.821 & $(0.50-2.40)$ & 1.01 & 0.990 & $(0.38-2.65)$ \\
\hline
\end{tabular}

${ }^{1}$ COR: Crude odds ratios. ${ }^{2}$ AOR: Odds ratios adjusted for region, gender, and relative family affluence. Boldface indicates statistically significant differences in $(p<0.05)$ odds for the

given substance use in the given group, as compared to the reference group. 


\subsection{Cannabis Use across Romantic Attraction}

In the univariate model, same- and both-gender attracted adolescents were significantly more likely, while those who had not been in love were significantly less likely to report cannabis use in the last 30 days compared to their opposite-gender attracted peers (Table 3). After adjusting for country/region, family affluence, and gender, a similar pattern was observed (the full model can be found in supplementary Table S4). Compared to those reporting opposite-gender love, those who had been in love with same-gender partners had odds of 2.2 and those in love with both-gender partners had odds of 3.6 (both significantly higher) of reporting cannabis use, while those who had never been in love had significantly lower odds $(\mathrm{AOR}=0.6)$; the odds of non-responders were not significantly different from their opposite-gender attracted peers. Same-gender attracted boys' odds were significantly higher $(\mathrm{AOR}=2.9)$ but same-gender attracted girls had similar odds $(\mathrm{AOR}=1.6)$ for cannabis use as their opposite-gender attracted peers. Both-gender attracted boys and girls had higher odds than those reporting opposite-gender attraction (boys' $\mathrm{AOR}=4.1$, girls' $\mathrm{AOR}=3.2$ ), whereas never having been in love was associated with significantly lower odds in both boys $(\mathrm{AOR}=0.7)$ and girls $(\mathrm{AOR}=0.5)$.

\subsection{Multiple Substance Use across Romantic Attraction}

The comparison of participants reporting multiple substance use among those with different patterns of attraction yielded analogous results to those found with use of single substances (Table 4). In both the univariate model and the model adjusted for country/region, family affluence and gender (the full model can be found in supplementary Table S5), same- and both-gender attracted adolescents had higher odds of using any two or all three substances than opposite-gender attracted young people, while not being in love was associated with significantly lower odds. Non-respondents had a similar likelihood to their opposite-gender attracted peers of reporting multiple substance use. However, in the gender-stratified analyses, only same-gender attracted boys had significantly higher odds of multiple substance use $(\mathrm{AOR}=2.1 ; p=0.02)$, while among girls, the difference was not statistically significant $(\mathrm{AOR}=1.4 ; p=0.16)$. When compared to opposite-gender attracted youth, both-gender attraction was associated with significantly higher odds of multiple substance use for both boys (AOR = 2.8) and girls (AOR = 2.2), while those not having been in love had significantly lower odds (boys' AOR = 0.5, girls' AOR $=0.4)$. Girls not responding to the love item also had significantly lower odds of multiple substance use $(\mathrm{AOR}=0.6, p=0.04)$, but not boys $(\mathrm{AOR}=0.9, p=0.65)$. 
Table 4. Crude and adjusted odds for multiple substance use, overall and by sex $(n=13,580)$.

\begin{tabular}{|c|c|c|c|c|c|c|c|c|c|c|c|c|}
\hline & \multirow{2}{*}{\multicolumn{3}{|c|}{ Univariate Model }} & \multirow{2}{*}{\multicolumn{3}{|c|}{ Multivariate Model (Overall) }} & \multicolumn{6}{|c|}{ Multivariate Model Stratified for Sex } \\
\hline & & & & & & & \multicolumn{3}{|c|}{ Boys $(n=6693)$} & \multicolumn{3}{|c|}{ Girls $(n=6811)$} \\
\hline & COR $^{1}$ & $p$ & $(95 \% \mathrm{CI})$ & $\mathrm{AOR}^{2}$ & $p$ & $(95 \% \mathrm{CI})$ & AOR & $p$ & $(95 \% \mathrm{CI})$ & AOR & $p$ & $(95 \% \mathrm{CI})$ \\
\hline \multicolumn{13}{|c|}{$\begin{array}{l}\text { Multiple substance use in the } \\
\text { last } 30 \text { days }{ }^{3}\end{array}$} \\
\hline Opposite-gender love & 1 & & & 1 & & & 1 & & & 1 & & \\
\hline Same-gender love & 1.79 & $<0.001$ & $(1.30-2.46)$ & 1.68 & 0.003 & $(1.20-2.35)$ & 2.12 & 0.002 & $(1.33-3.64)$ & 1.39 & 0.161 & $(0.88-2.20)$ \\
\hline Both-gender love & 2.34 & $<0.001$ & $(1.77-3.09)$ & 2.43 & $<0.001$ & $(1.79-3.31)$ & 2.78 & $<0.001$ & $(1.64-4.70)$ & 2.24 & $<0.001$ & $(1.54-3.27)$ \\
\hline Not in love & 0.47 & $<0.001$ & $(0.39-0.56)$ & 0.44 & $<0.001$ & $(0.36-0.53)$ & 0.53 & $<0.001$ & $(0.40-0.70)$ & 0.38 & $<0.001$ & $(0.29-0.49)$ \\
\hline Not responding & 0.84 & 0.291 & $(0.60-1.16)$ & 0.74 & 0.081 & $(0.53-1.03)$ & 0.90 & 0.651 & $(0.58-1.41)$ & 0.59 & 0.044 & $(0.35-0.99)$ \\
\hline
\end{tabular}

${ }^{1}$ COR: Crude odds ratios. ${ }^{2}$ AOR: Odds ratios adjusted for region, gender, and relative family affluence. Boldface indicates statistically significant differences in $(p<0.05)$ odds for the

given substance use in the given group, as compared to the reference group. ${ }^{3}$ Based on cigarettes, alcohol or cannabis in the last 30 days. 


\section{Discussion}

This study aimed to explore the association between different romantic attraction patterns and substance use across national representative samples of adolescents from eight European countries and regions with various geographical location, history and level of tolerance towards sexual minorities. Our findings indicate higher risks for both same- and both-gender attracted youth to engage in substance use. This pattern was observed for single (cigarette smoking, alcohol consumption, drunkenness, and cannabis use) and multiple substance use (any two or more of cigarette smoking, alcohol consumption, or cannabis use), thus supporting findings from existing international literature. The fact that the pattern of the odds ratios remained very similar after controlling for gender, country/region and relative family affluence suggests that the elevated vulnerability of SMY to be engaged in substance use is a universal phenomenon, at least across the eight investigated European countries. These findings imply that as well as differentiating between heterosexual and homosexual or bisexual orientation, separating monosexual (heterosexual or gay/lesbian) and plurisexual (bisexual) identities may also reveal health disparities. Expanding the investigation to those who have not been in love revealed that, to a certain extent, reporting being in love (irrespective the gender of the love partner) was associated with higher odds of substance use than not having been in love. These results can be integrated with the minority stress and romantic stress theoretical models.

\subsection{Gender and Attraction}

Differences in alcohol, drunkenness, cannabis and multiple substance use were found across the groups of same- and both-gender attracted boys and girls. Regarding alcohol and drunkenness, same-gender attracted boys were at somewhat (but not to a significant extent) higher risk than both-gender attracted boys. Among girls, both-gender attraction was associated with higher risk for all five substance use indicators compared to same-gender attraction. This finding is in line with other studies showing that bisexual or both-gender attracted youths are among the highest risk of all SMY groups in relation to substance use [8,20,24], and even they are not homogenous in terms of risk [57].

\subsection{Socioeconomic Status, Country and Attraction}

Both family affluence and country/region were significant predictors in all multivariate models, however both unadjusted and adjusted substance use models follow very similar patterns. This indicates that the association between romantic attraction and substance use is not substantially influenced by family background or country/region of residence, at least in the eight European countries featured in our study. While lower socioeconomic status is associated with greater likelihood of reporting substance use [58], some argue that minority stress exacerbates the involvement of all SMYs in substance use, against which racial (or socio-economic) factors are not protective [11]. In other words, sexual minority status may be more strongly associated with substance use than socio-economic status. The observation that the risks of substance use among SMY is similar across regions and countries reinforces existing findings in this area, from single European countries [59-63] or from cross-cultural investigations [21].

\subsection{Love: A 'Sweet Poison'?}

In line with our hypotheses, we have observed that those young people who reported never having been in love had significantly lower (0.4-0.7 times) odds of any single or multiple substance use than their opposite-gender attracted peers. This was found in the general models as well as in those disaggregated for gender. In other words, it seems that never having been in love is protective against substance use. This finding is in line with available evidence that romantic relationships (irrespective of the gender of the partner) may be associated with stress in adolescence [64], thus support the concept of romantic stress. Involvement in romantic relationships may be stressful for young people for a variety of reasons. These include separation and individuation from family, cultural expectations, conflicts 
with the romantic partner, and a double standard of love and sexual initiation for boys and girls [41,65]. As mentioned in Section 1.4, some participants might have been engaged in substance use (e.g., alcohol and drug consumption) to cope with romantic stress [41]. The romantic partner's substance use habits may also be predictive of the adolescents' own substance use [66]. Time spent with peers is predictive of substance use [67], and a similar association might be there with romantic partners. Further studies are needed to disentangle the separate effects of minority and romantic stress in SMY.

\subsection{Non-Responders}

Not responding to the item on love was not associated with higher odds of cigarette smoking, drunkenness, and cannabis use, but was associated with lower odds for alcohol use (in both boys and girls) and for multiple substance use (in girls) than for those who reported opposite-gender attraction. Adolescents may have various motives for not answering survey questions related to sexual orientation. They may be reluctant or unwilling to assume a socially stigmatized identity label, or it may reflect personal, cultural, religious, or political resistance to being categorized or defined by their sexuality [42]. We do not know participants' motives for not responding to the item, but we speculate that this is not associated with elevated stress (based on the above-mentioned findings on the association of stress and frequent substance use). When developing the item on love, a few young people told our research team that they felt it is too private [68]. Further qualitative studies are needed to better understand young people's motivations for not answering questions of this nature.

\subsection{Limitations}

Our findings are limited by the fact that the study was cross-sectional, therefore no causal or temporal inferences can be made. Given the low subsample sizes in countries, we could not carry out country-stratified analyses. However, from the fact that country as a control variable (as gender and family affluence) did not substantially change the pattern of the results, we infer that SMY may be at elevated risk regardless of their gender, family background and country of residence, at least in these eight European countries/regions.

We have concentrated on feelings of love for opposite-, same- or both-gender partners, which may not totally correspond to self-identified sexual orientation, erotic desire or sexual behavior [42,43].

Finally, we used a binary variable (boy or girl) to categorize adolescents' gender, which does not reflect trans, non-binary or other gender minority groups. The links between gender, biological sex and sexual orientation constitute a very complex issue [69]. The HBSC International Network is currently working on how the survey can be more inclusive of both gender and sex diversity.

\subsection{Reducing Risk and Promoting Resilience in Sexual Minority Youth}

How can we reduce the risk associated with romantic attraction (compared to those who have not been in a romantic relationship) and the risk associated with both- and same-gender attraction compared to those who are attracted to opposite-gender partners? Love is experienced by many young people; professionals working with adolescents in health or social care, or educational settings, should be aware of the potential stressful effects of romantic relationships and minority stress, and be prepared to discuss these intimate matters with young people. Promoting healthy romantic relationships, both for SMY and heterosexual youth, may reduce (romantic) stress and have a positive impact on peer norms. Direct measures to promote health and resilience in SMY, such as 'gay-straight alliances' or 'gender-sexuality alliances' [70], media-based interventions to address sexual orientation related prejudice [71] or introducing safe school policies [72] have a documented beneficial effect on the health of not just SMY, but on heterosexual adolescents as well. Risk prevention and enhancing resilience and well-being in SMY should be part of national youth health strategies [73]. Some suggest that researchers and practitioners should consider how to shift from a victimizing and pathologizing narrative, which describes sexual minority individuals as 'vulnerable' [74]. A more positive view on sexual (and gender) minority people include, for instance, resilience, compassion, and tolerance towards members of 
other minorities [75]. Despite the hardships sexual minority young people experience, they have the potential to express their identity and love and lead healthy and happy lives.

\subsection{Future Directions}

Dimensions of sexual orientation, involvement in romantic attractions, minority stress, risk behaviors and psychosocial factors constitute a complex causal 'web'. Future studies are needed to map how bullying involvement and social support shape substance use and other risky and health promoting behaviors, and various health outcomes in SMY. Using a positive approach, such research projects may also map health-protective factors and resources in sexual minority youth.

\section{Conclusions}

Sexual minority youth from eight European countries (identified based on reporting love for same- or both-gender partners) were found to be at higher risk of substance use behaviors than their opposite-gender attracted peers. On the other hand, adolescents who reported not having been in love were at lower risk of these substance use behaviors. These results support the assertion that romantic experiences on their own might be stressful for adolescents across different cultures, and that sexual minority status is associated with higher risk of substance use even after controlling for country/region, socio-economic status and gender. Targeted policy actions are needed to reduce risk and promote well-being and resilience in SMY, and further cross-national research needs to be conducted to better understand how dimensions of sexual orientation impact the health of young people.

Supplementary Materials: The following are available online at http:/www.mdpi.com/1660-4601/16/17/3063/ s1. Data collected in the 2013/2104 round of HBSC can be accessed following registration at https://www. uib.no/en/hbscdata. The supplementary tables can be downloaded from: https://osf.io/d38px/?view_only= 690caa0ccc3545eea8e7c32cf93f41fc.

Author Contributions: Conceptualization, A.K.; data curation, A.K.; formal analysis, A.K.; funding acquisition, E.B.T., E.M.S. and S.N.G.; investigation, A.K., A.C., H.Y., N.M., D.P., R.T., E.B.T., A.V., E.M.S. and S.N.G.; methodology, A.K., A.C., N.M., D.P., R.T., E.B.T. and A.V.; project administration, A.K.; supervision, E.M.S. and S.N.G.; validation, A.K., A.C., H.Y., N.M., D.P., R.T., A.V. and S.N.G.; writing-original draft, A.K., D.P., R.T. and A.V.; writing—review \& editing, A.K., A.C., H.Y., N.M., E.B.T., E.M.S. and S.N.G.

Funding: HBSC Belgium (FWB) is funded by the Wallonia-Brussels Federation (FWB), the Office of Birth and Childhood (ONE), the Walloon Region and the Brussels-Capital Region. HBSC Bulgaria is funded by UNICEF-Bulgaria. HBSC England is funded by the Department of Health and Social Care. HBSC France is funded by Santé Publique France and OFDT (French Monitoring Centre for Drug Use and Addiction). HBSC Hungary is funded by ELTE Eötvös Loránd University. HBSC Iceland is funded by grants from the Icelandic Directorate of Health, KEA and the University of Akureyri. North Macedonia is funded by the United Nations Population Fund/United Nations Development Programme. HBSC Switzerland is funded by the Swiss Federal Office of Public Health and most of the Swiss cantons. The study was funded in part by grant \#FDN 154335 from the Canadian Institutes of Health Research (Saewyc, PI).

Acknowledgments: HBSC is an international study carried out in collaboration with WHO Europe. The International Coordinator of the 2013/2014 survey was Joanna Inchley (University of Glasgow, Scotland) and the Data Bank Manager was Oddrun Samdal (University of Bergen, Norway). The following Principal Investigators of national HBSC Teams gave us permission to use national data from the given country: Katia Castetbon (French Belgium), Lidiya Vasileva (Bulgaria), Fiona Brooks and Ellen Klemera (England), Emmanuelle Godeau (France), Lina Kostarova-Unkovska (North Macedonia), Ágnes Németh (Hungary), Arsaell Arnarsson (Iceland), and Marina Delgrande Jordan and Hervé Kuendig (Switzerland). We are grateful for HBSC Communications Officer Joseph Hancock and our colleagues in the HBSC Sexual Health Focus Group for their help. For details on HBSC, see http://www.hbsc.org.

Conflicts of Interest: The authors declare no conflict of interest.

\section{References}

1. Semlyen, J.; King, M.; Varney, J.; Hagger-Johnson, G. Sexual orientation and symptoms of common mental disorder or low wellbeing: Combined meta-analysis of 12 UK population health surveys. BMC Psychiatry 2016, 16, 67. [CrossRef] [PubMed] 
2. Hafeez, H.; Zeshan, M.; A Tahir, M.; Jahan, N.; Naveed, S. Health Care Disparities Among Lesbian, Gay, Bisexual, and Transgender Youth: A Literature Review. Cureus 2017, 9, e1184. [CrossRef] [PubMed]

3. Marshal, M.P.; Friedman, M.S.; Stall, R.; King, K.M.; Miles, J.; Gold, M.A.; Bukstein, O.G.; Morse, J.Q. Sexual orientation and adolescent substance use: A meta-analysis and methodological review. Addiction 2008, 103, 546-556. [CrossRef] [PubMed]

4. Goldbach, J.; Tanner-Smith, E.; Bagwell, M.; Dunlap, S. Minority stress and substance use in sexual minority adolescents: A meta-analysis. Prev. Sci. 2014, 15, 350-363. [CrossRef] [PubMed]

5. Austin, S.B.; Ziyadeh, N.; Fisher, L.B.; Kahn, J.A.; Colditz, G.A.; Frazier, A.L. Sexual Orientation and Tobacco Use in a Cohort Study of US Adolescent Girls and Boys. Arch. Pediatr. Adolesc. Med. 2004, 158, 317-322. [CrossRef]

6. Dai, H. Tobacco Product Use among Lesbian, Gay, and Bisexual Adolescents. Pediatrics 2017, 139, e20163276. [CrossRef]

7. Lee, J.; Griffin, G.K.; Melvin, C.L. Tobacco use among sexual minorities in the USA, 1987 to May 2007: A systematic review. Tob. Control 2009, 18, 275-282. [CrossRef]

8. Corliss, H.L.; Rosario, M.; Birkett, M.A.; Newcomb, M.E.; Buchting, F.O.; Matthews, A.K. Sexual Orientation Disparities in Adolescent Cigarette Smoking: Intersections With Race/Ethnicity, Gender, and Age. Am. J. Public Health 2014, 104, 1137-1147. [CrossRef]

9. Watson, R.J.; Lewis, N.M.; Fish, J.N.; Goodenow, C. Sexual minority youth continue to smoke cigarettes earlier and more often than heterosexuals: Findings from population-based data. Drug Alcohol Depend. 2018, 184, 64-70. [CrossRef]

10. Fish, J.N.; Turner, B.; Phillips, G.; Russell, S.T. Cigarette Smoking Disparities between Sexual Minority and Heterosexual Youth. Pediatr 2019, 143, e20181671. [CrossRef]

11. Newcomb, M.E.; Heinz, A.J.; Birkett, M.; Mustanski, B. A Longitudinal Examination of Risk and Protective Factors for Cigarette Smoking among Lesbian, Gay, Bisexual and Transgender Youth. J. Adolesc. Health 2014, 54, 558-564. [CrossRef] [PubMed]

12. Rosario, M.; Schrimshaw, E.W.; Hunter, J. Cigarette smoking as a coping strategy: Negative implications for subsequent psychological distress among lesbian, gay, and bisexual youths. J. Pediatr. Psychol. 2011, 36, 731-742. [CrossRef] [PubMed]

13. Hatzenbuehler, M.; Jun, H.J.; Corliss, H.; Austin, S. Structural stigma and cigarette smoking in a prospective cohort study of sexual minority and heterosexual youth. Ann. Behav. Med. 2014, 47, 48-56. [CrossRef] [PubMed]

14. Talley, A.E.; Hughes, T.L.; Aranda, F.; Birkett, M.; Marshal, M.P. Exploring Alcohol-Use Behaviors among Heterosexual and Sexual Minority Adolescents: Intersections with Sex, Age, and Race/Ethnicity. Am. J. Public Health 2014, 104, 295-303. [CrossRef] [PubMed]

15. Garofalo, R.; Wolf, R.C.; Kessel, S.; Palfrey, J.; Durant, R.H. The Association between Health Risk Behaviors and Sexual Orientation Among a School-based Sample of Adolescents. Pediatrics 1998, 101, 895-902. [CrossRef] [PubMed]

16. Fish, J.N.; Pasley, K. Sexual (Minority) Trajectories, Mental Health, and Alcohol Use: A Longitudinal Study of Youth as They Transition to Adulthood. J. Youth Adolesc. 2015, 44, 1508-1527. [CrossRef] [PubMed]

17. Donath, C.; Gräßel, E.; Baier, D.; Pfeiffer, C.; Karagülle, D.; Bleich, S.; Hillemacher, T. Alcohol consumption and binge drinking in adolescents: Comparison of different migration backgrounds and rural vs. urban residence-A representative study. BMC Public Health 2011, 11, 84. [CrossRef] [PubMed]

18. Vieno, A.; Altoè, G.; Kuntsche, E.; Elgar, F.J. Do public expenditures on health and families relate to alcohol abstaining in adolescents? Multilevel study of adolescents in 24 countries. Drug Alcohol Rev. 2018, 37, S120-S128. [CrossRef]

19. Fish, J.N.; Baams, L. Trends in Alcohol-Related Disparities between Heterosexual and Sexual Minority Youth from 2007 to 2015: Findings from the Youth Risk Behavior Survey. LGBT Health 2018, 5, 359-367. [CrossRef]

20. Talley, A.E.; Turner, B.; Foster, A.M.; Phillips, G. Sexual Minority Youth at Risk of Early and Persistent Alcohol, Tobacco, and Marijuana Use. Arch. Sex. Behav. 2019, 48, 1073-1086. [CrossRef]

21. Demant, D.; Hides, L.; White, K.M.; Kavanagh, D.J. LGBT communities and substance use in Queensland, Australia: Perceptions of young people and community stakeholders. PLoS ONE 2018, 13, e0204730. [CrossRef] [PubMed] 
22. Gorbach, P.M.; Javanbakht, M.; Shover, C.L.; Bolan, R.K.; Ragsdale, A.; Shoptaw, S. Associations Between Cannabis Use, Sexual Behavior, and Sexually Transmitted Infections/Human Immunodeficiency Virus in a Cohort of Young Men Who Have Sex With Men. Sex. Transm. Dis. 2019, 46, 105-111. [CrossRef] [PubMed]

23. Goldbach, J.T.; Schrager, S.M.; Dunlap, S.L.; Holloway, I.W. The application of minority stress theory to marijuana use among sexual minority adolescents. Subst. Use Misuse 2015, 50, 366-375. [CrossRef] [PubMed]

24. Blais, M.; Bergeron, F.A.; Duford, J.; Boislard, M.A.; Hebert, M. Health outcomes of sexual-minority youth in Canada: An overview. Adolesc. Saude 2015, 12, 53-73. [PubMed]

25. Watson, R.J.; Goodenow, C.; Porta, C.; Adjei, J.; Saewyc, E. Substance use among sexual minorities: Has it actually gotten better? Subst. Use Misuse 2018, 53, 1221-1228. [CrossRef] [PubMed]

26. Goldbach, J.; Fisher, B.W.; Dunlap, S. Traumatic Experiences and Drug Use by LGB Adolescents: A Critical Review of Minority Stress. J. Soc. Work. Pract. Addict. 2015, 15, 90-113. [CrossRef]

27. Newcomb, M.E.; Ryan, D.T.; Greene, G.J.; Garofalo, R.; Mustanski, B. Prevalence and Patterns of Smoking, Alcohol Use, and Illicit Drug Use in Young Men Who Have Sex with Men. Drug Alcohol Depend. 2014, 141, 65-71. [CrossRef] [PubMed]

28. Marshal, M.P.; King, K.M.; Stepp, S.D.; Hipwell, A.; Smith, H.; Chung, T.; Friedman, M.S.; Markovic, N. Trajectories of alcohol and cigarette use among sexual minority and heterosexual girls. J. Adolesc. Health 2012, 50, 97-99. [CrossRef]

29. Lea, T.; Reynolds, R.; de Wit, J. Alcohol and club drug use among same-sex attracted young people: Associations with frequenting the lesbian and gay scene and other bars and nightclubs. Subst. Use Misuse 2013, 48, 129-136. [CrossRef]

30. Balsam, K.F.; Molina, Y.; Lehavot, K. Alcohol and Drug Use in Lesbian, Gay, Bisexual, and Transgender (LGBT) Youth and Young Adults. In Principles of Addiction; Elsevier: Amsterdam, The Netherlands, 2013; pp. 563-573.

31. Matthews, D.D.; Blosnich, J.R.; Farmer, G.W.; Adams, B.J. Operational definitions of sexual orientation and estimates of adolescent health risk behaviors. LGBT Health 2014, 1, 42-49. [CrossRef]

32. Meyer, I.H. Prejudice, Social Stress, and Mental Health in Lesbian, Gay, and Bisexual Populations: Conceptual Issues and Research Evidence. Psychol. Bull. 2003, 129, 674-697. [CrossRef] [PubMed]

33. Meyer, I.H. Prejudice and Discrimination as Social Stressors. In The Health of Sexual Minorities; Springer Science and Business Media: New York, NY, USA, 2007; pp. 242-267.

34. Hatzenbuehler, M.L. How Does Sexual Minority Stigma "Get Under the Skin"? A Psychological Mediation Framework. Psychol. Bull. 2009, 135, 707-730. [CrossRef] [PubMed]

35. Lea, T.; De Wit, J.; Reynolds, R. Minority Stress in Lesbian, Gay, and Bisexual Young Adults in Australia: Associations with Psychological Distress, Suicidality, and Substance Use. Arch. Sex. Behav. 2014, 43, 1571-1578. [CrossRef] [PubMed]

36. Hughes, T.L.; Wilsnack, S.C.; Kantor, L.W. The influence of gender and sexual orientation on alcohol use and alcohol-related problems: Toward a global perspective. Alcohol Res. 2016, 38, 121-132. [PubMed]

37. Saewyc, E.M. Research on adolescent sexual orientation: Development, health disparities, stigma and resilience. J. Res. Adolesc. 2011, 21, 256-272. [CrossRef]

38. Bränström, R.; van der Star, A. More knowledge and research concerning the health of lesbian, gay, bisexual and transgender individuals is needed. Eur. J. Public Health 2016, 26, 208-209. [CrossRef]

39. ILGA-Europe. Annual Review of the Human Rights Situation of Lesbian, Gay, Bisexual, Trans and Intersex People in Europe; ILGA-Europe: Brussels, Belgium, 2014.

40. Demant, D.; Hides, L.; Kavanagh, D.J.; White, K.M.; Winstock, A.R.; Ferris, J. Differences in substance use between sexual orientations in a multi-country sample: Findings from the Global Drug Survey 2015. J. Public Health (Oxf.) 2017, 39, 532-541. [CrossRef]

41. Seiffge-Krenke, I.; Bosma, H.; Chau, C.; Çok, F.; Gillespie, C.; Loncaric, D.; Molinar, R.; Cunha, M.; Veisson, M.; Rohail, I. All they need is love? Placing romantic stress in the context of other stressors: A 17-nation study. Int. J. Behav. Dev. 2010, 34, 106-112. [CrossRef]

42. Savin-Williams, R.C.; Cohen, K.M. Development of Same-Sex Attracted Youth. In The Health of Sexual Minorities: Public Health Perspectives on Lesbian, Gay, Bisexual and Transgender Populations; Meyer, I.H., Northridge, M.E., Eds.; Springer US: Boston, MA, USA, 2007; pp. 27-47. 
43. Geary, R.S.; Tanton, C.; Erens, B.; Clifton, S.; Prah, P.; Wellings, K.; Mitchell, K.R.; Datta, J.; Gravningen, K.; Fuller, E.; et al. Sexual identity, attraction and behaviour in Britain: The implications of using different dimensions of sexual orientation to estimate the size of sexual minority populations and inform public health interventions. PLoS ONE 2018, 13, e0189607. [CrossRef]

44. Calzo, J.P.; Blashill, A.J. Child Sexual Orientation and Gender Identity in the Adolescent Brain Cognitive Development Cohort Study. JAMA Pediatr. 2018, 172, 1090-1092. [CrossRef]

45. Költő, A.; Young, H.; Burke, L.; Moreau, N.; Cosma, A.; Magnusson, J.; Windlin, B.; Reis, M.; Saewyc, E.M.; Godeau, E.; et al. Love and Dating Patterns for Same- and Both-Gender Attracted Adolescents Across Europe. J. Res. Adolesc. 2018, 28, 772-778. [CrossRef] [PubMed]

46. Russell, S.T.; Driscoll, A.K.; Truong, N. Adolescent Same-Sex Romantic Attractions and Relationships: Implications for Substance Use and Abuse. Am. J. Public Health 2002, 92, 198-202. [CrossRef] [PubMed]

47. Marshal, M.P.; Friedman, M.S.; Stall, R.; Thompson, A.L. Individual trajectories of substance use in lesbian, gay and bisexual youth and heterosexual youth. Addiction 2009, 104, 974-981. [CrossRef] [PubMed]

48. Diamond, L.M. What does sexual orientation orient? A biobehavioral model distinguishing romantic love and sexual desire. Psychol. Rev. 2003, 110, 173-192. [CrossRef] [PubMed]

49. Inchley, J.; Currie, D.; Young, T.; Samdal, O.; Torsheim, T.; Augustson, L.; Mathisen, F.; Aleman-Diaz, A.; Molcho, M.; Weber, M.; et al. Growing up Unequal: Gender and Socioeconomic Differences in Young people's Health and Well-Being. In Health Behaviour in School-Aged Children (HBSC) Study. International Report from the 2013/2014 Survey; WHO Regional Office for Europe: Copenhagen, Denmark, 2016.

50. Health Behaviour in School-Aged Children (HBSC). Study Protocol: Background, Methodology and Mandatory Items for the 2013/14 Survey; Currie, C., Inchley, J., Molcho, M., Lenzi, M., Veselska, Z., Wild, F., Eds.; Child and Adolescent Health Research Unit (CAHRU), University of St. Andrews: St. Andrews, UK, 2014.

51. Guttormsson, U.; Leifman, H.; Kraus, L.; Molinaro, S.; Monshouwer, K.; Trapencieres, M.; Vincente, J.; Englund, A.; Svensson, J. ESPAD 2015: Methodology; European Monitoring Centre for Drugs and Drug Addiction (EMCDDA) and The European School Survey Project on Alcohol and Other Drugs (ESPAD): Lisbon, Portugal, 2016.

52. Currie, C.; Molcho, M.; Boyce, W.; Holstein, B.; Torsheim, T.; Richter, M. Researching health inequalities in adolescents: The development of the Health Behaviour in School-Aged Children (HBSC) Family Affluence Scale. Soc. Sci. Med. 2008, 66, 1429-1436. [CrossRef] [PubMed]

53. Schnohr, C.; Makransky, G.; Kreiner, S.; Torsheim, T.; Hofmann, F.; De Clercq, B.; Elgar, F.; Currie, C. Item response drift in the Family Affluence Scale: A study on three consecutive surveys of the Health Behaviour in School-aged Children (HBSC) survey. Measurement 2013, 46, 3119-3126. [CrossRef]

54. Torsheim, T.; Cavallo, F.; Levin, K.A.; Schnohr, C.; Mazur, J.; Niclasen, B.; Currie, C.; The FAS Development Study Group. Psychometric validation of the revised family affluence scale: A latent variable approach. Child Indic. Res. 2016, 9, 771-784. [CrossRef]

55. Allison, P.D. Measures of Fit for Logistic Regression. In Proceedings of the SAS Global Forum, Washington, DC, USA, 23-26 March 2014.

56. Barrett, P. Structural equation modelling: Adjudging model fit. Pers. Individ. Differ. 2007, 42, 815-824. [CrossRef]

57. Feinstein, B.A.; Turner, B.C.; Beach, L.B.; Korpak, A.K.; Phillips, G. Racial/Ethnic Differences in Mental Health, Substance Use, and Bullying Victimization Among Self-Identified Bisexual High School-Aged Youth. LGBT Health 2019, 6, 174-183. [CrossRef]

58. Adler, N.E.; Boyce, T.; Chesney, M.A.; Cohen, S.; Folkman, S.; Kahn, R.L.; Syme, S.L. Socioeconomic status and health: The challenge of the gradient. Am. Psychol. 1994, 49, 15-24. [CrossRef]

59. Arnarsson, A.; Sveinbjornsdottir, S.; Thorsteinsson, E.B.; Bjarnason, T. Suicidal risk and sexual orientation in adolescence: A population-based study in Iceland. Scand. J. Public Health 2015, 43, 497-505. [CrossRef] [PubMed]

60. Thorsteinsson, E.B.; Loi, N.M.; Sveinbjornsdottir, S.; Arnarsson, A. Sexual orientation among Icelandic year 10 adolescents: Changes in health and life satisfaction from 2006 to 2014. Scand. J. Psychol. 2017, 58, 530-540. [CrossRef] [PubMed]

61. Clarke, A.; Beenstock, J.; Lukacs, J.N.; Turner, L.; Limmer, M. Major risk factors for sexual minority young people's mental and physical health: Findings from a county-wide school-based health needs assessment. J. Public Health (Oxf.) 2018, fdy167. [CrossRef] [PubMed] 
62. Amos, R.; Manalastas, E. Mental Health, Social Adversity and Health Outcomes in Sexual Minority Adolescents Aged 14 Years: Findings from the Millennium Cohort Study (MCS6). In Proceedings of the BPS Psychology of Sexualities Annual Conference, London, UK, 4-5 July 2019.

63. Kuyper, L.; De Roos, S.; Iedema, J.; Stevens, G. Growing Up With the Right to Marry: Sexual Attraction, Substance Use, and Well-Being of Dutch Adolescents. J. Adolesc. Health 2016, 59, 276-282. [CrossRef] [PubMed]

64. Collins, W.A.; Welsh, D.P.; Furman, W. Adolescent romantic relationships. Annu. Rev. Psychol. 2008, 60, 631-652. [CrossRef] [PubMed]

65. Moreau, N.; Költő, A.; Young, H.; Maillochon, F.; Godeau, E. Negative feelings about the timing of first sexual intercourse: Findings from the Health Behaviour in School-aged Children study. Int. J. Public Health 2019, 64, 219-227. [CrossRef] [PubMed]

66. Aikins, J.W.; Simon, V.A.; Prinstein, M.J. Romantic Partner Selection and Socialization of Young Adolescents' Substance Use and Behavior Problems. J. Adolesc. 2010, 33, 813-826. [CrossRef] [PubMed]

67. Barnes, G.M.; Hoffman, J.H.; Welte, J.W.; Farrell, M.P.; Dintcheff, B.A. Adolescents' time use: Effects on substance use, delinquency and sexual activity. J. Youth Adolesc. 2007, 36, 697-710. [CrossRef]

68. Young, H.; Költő, A.; Reis, M.; Saewyc, E.M.; Moreau, N.; Burke, L.; Cosma, A.; Windlin, B.; Gabhainn, S.N.; Godeau, E. Sexual health questions included in the Health Behaviour in School-aged Children (HBSC) study: An international methodological pilot investigation. BMC Med. Res. Methodol. 2016, 16, 169. [CrossRef]

69. Fausto-Sterling, A. Gender/Sex, Sexual Orientation, and Identity Are in the Body: How Did They Get There? J. Sex Res. 2019, 56, 529-555. [CrossRef]

70. Li, G.; Wu, A.D.; Marshall, S.K.; Watson, R.J.; Adjei, J.K.; Park, M.; Saewyc, E.M. Investigating site-level longitudinal effects of population health interventions: Gay-Straight Alliances and school safety. SSM Popul. Health 2019, 7, 100350. [CrossRef] [PubMed]

71. Burk, J.; Park, M.; Saewyc, E.M. A Media-Based School Intervention to Reduce Sexual Orientation Prejudice and Its Relationship to Discrimination, Bullying, and the Mental Health of Lesbian, Gay, and Bisexual Adolescents in Western Canada: A Population-Based Evaluation. Int. J. Environ. Res. Public Health 2018, 15, 2447. [CrossRef] [PubMed]

72. Russell, S.T.; Kosciw, J.; Horn, S.; Saewyc, E.M. Safe schools policy for LGBTQ students. SRCD Soc. Policy Rep. 2010, 24, 1-25. [CrossRef]

73. DCYA. LGBTI+ National Youth Strategy 2018-2020; Department of Children and Youth Affairs: Dublin, Ireland, 2018.

74. Fineman, M.A. Vulnerability, Resilience, and LGBT Youth Symposium: LGBT youth: Reconciling pride, family, and community. Temple Political Civ. Rights Law Rev. 2013, 23, 307-330.

75. Riggle, E.D.B.; Rostosky, S.S. A Positive View of LGBTQ: Embracing Identity and Cultivating Well-Being; Rowman \& Littlefield Publishers, Inc.: Lanham, MD, USA, 2012.

(C) 2019 by the authors. Licensee MDPI, Basel, Switzerland. This article is an open access article distributed under the terms and conditions of the Creative Commons Attribution (CC BY) license (http://creativecommons.org/licenses/by/4.0/). 